\title{
Fragebogen
}

1 Welche klinischen Merkmale sprechen für einen epileptischen Anfall und gegen eine Synkope?

A Myoklonien

B Stuhl- und Harninkontinenz

C Reorientierungsphase von 3 Minuten

D Zungenbiss

E Reorientierungsphase von 20 Minuten

2 Welche Aussage trifft nicht zu? Nennen Sie neurologische Erkrankungen, bei denen es gehäuft zu Synkopen kommen kann

A Morbus Parkinson

B Polyneuropathie

C Multiple Sklerose

D Syringomyelie

E Multisystematrophie

3 Welche Aussage trifft zu?

Nach einem ersten epileptischen

Anfall liegt das Risiko einen zweiten

zu erleiden über 5 Jahre bei

A zirka $15 \%$

B zirka $25 \%$

C zirka $35 \%$

D zirka $45 \%$

E zirka $55 \%$

4 Welche Aussage zur Subarachnoidalblutung (SAB) trifft nicht zu?

A Leitsymptom der $\mathrm{SAB}$ ist der typische Vernichtungskopfschmerz

B Eine $S A B$ geht stets mit Bewusstseinsstörungen einher

C Vegetative Begleitsymptome (z. B. Übelkeit und Erbrechen) sind häufig

D Ursache kann ein rupturiertes Aneurysma der hirnversorgenden Gefäße sein

E Eine $S A B$ ist ein neurologischer Notfall

5 Welche Aussage zu symptomatischen Kopfschmerzen trifft zu?

A Bei einer bakteriellen Meningitis kann auf eine Lumbalpunktion zur Liquorentnahme verzichtet werden
B Ein epileptischer Anfall ist nie Zeichen einer intracraniellen Blutung

C Die sogenannte "Sturzsenkung“ in Kombination mit schläfenbetonten Kopfschmerzen ist verdächtig auf eine Arteriitis temporalis

D Neu aufgetretene Kopfschmerzen sind praktisch immer harmlos

E Acetylsalicylsäure wird bei intracraniellen Blutungen zur Schmerztherapie eingesetzt

6 Welche Aussage trifft nicht zu?

A Sinusvenenthrombosen treten häufig in der Schwangerschaft auf

B Die Computertomografie ist eines der wichtigsten diagnostischen Methoden im Rahmen der Kopfschmerzabklärung

C Eine sogenannte „Warnblutung“ kann ein schmerzfreies Intervall nach sich ziehen

D Halbseitensymptome können Ausdruck einer intracraniellen Blutung sein

E Ein Meningismus tritt nur bei Hirnhautentzündungen auf

7 Welche Antwort zum GuillainBarré-Syndrom ist richtig?

A Initial fallen motorische Defizite an den Armen auf

B Häufigster betroffener Hirnnerv ist der N. glossopharyngeus

C Sensible Defizite stehen im Vordergrund der Erkrankung

D Manche Patienten werden intubationspflichtig

E Die meisten Patienten genesen vollständig

8 Welche Antwort ist falsch?

A Eine Therapiemöglichkeit ist die Gabe von Immunglobulinen

B Eine Therapie ist indiziert ab einer freien Gehstrecke von weniger als fünf Metern

C Mittels Plasmapherese kann der Krankheitsverlauf abgekürzt werden
D Mittels Glukokortikoiden kann der Krankheitsverlauf abgekürzt werden

E Bei schnellem Krankheitsprogress ist eine Behandlungsindikation gegeben

9 Welche Antwort ist richtig?

A Vorwiegend nächtliche Schmerzen können auf eine spezifische Ursache der Schmerzen, z. B. einen Morbus Bechterew, hindeuten

B Das Cauda-equina-Syndrom besteht aus der Trias Sensibilitätsstörungen im Oberschenkel, Fußheberparese und kleinschrittigem Gangbild

C In den ersten vier Wochen nach Beschwerdebeginn sollte bei unspezifischen Beschwerden die komplette Diagnostik abgeschlossen sein

D Arbeitsplatzunzufriedenheit steht in keinerlei Verbindung zur Entwicklung chronischer Rückenschmerzen

E Durchhaltestrategien sind günstig für die Prognose von Rückenschmerzen

10 Welche Antwort ist richtig?

A In der Frühphase sollte Aktivität weitgehend vermieden werden

B Paracetamol und NSAR sowie gegebenenfalls Muskelrelaxanzien stellen die medikamentöse Basis in der Behandlung von akuten Rückenschmerzen dar

C Frühe Krankengymnastik sowie epidurale Applikation von Steroiden spielen in der Behandlung von akuten Rückenschmerzen eine große Rolle

D Warnzeichen können nur in der Frühphase auftreten, eine Symptomkontrolle bei Beschwerdepersistenz ist nicht erforderlich

E Erst bei Schmerzen, die länger als ein Jahr anhalten, muss nach Risikofaktoren für eine Chronifizierung gesucht werden 
Antwortbogen online-Teilnahme unter http://cme.thieme.de

\begin{tabular}{lllllllllllllll}
$\begin{array}{l}\text { Lernerfolgskontrolle } \\
\text { (nur eine Antwort pro Frage ankreuzen) }\end{array}$ & 1 & A & B & C & D & E & 6 & A & B & C & D & E \\
& 2 & A & B & C & D & E & 7 & A & B & C & D & E \\
\hline & 3 & A & B & C & D & E & $\mathbf{8}$ & A & B & C & D & E \\
\hline & 4 & A & B & C & D & E & $\mathbf{9}$ & A & B & C & D & E \\
\hline 5 & A & B & C & D & E & $\mathbf{1 0}$ & A & B & C & D & E
\end{tabular}

Ihre Adresse

Name $\quad$ Vorname

Straße

PLZ, Ort

Erklärung

Ich versichere, dass ich die Beantwortung der Fragen selbst und ohne fremde Hilfe durchgeführt habe.

Ort/Datum

Unterschrift

CME-Wertmarken

für Nicht-Abonnenten
CME-Wertmarken (für Teilnehmer, die die Notfall \& Hausarztmedizin nicht abonniert haben) können beim Verlag zu folgenden Bedingungen erworben werden: 6er-Pack Thieme-CME-Wertmarken, Preis: 63,- Euro inkl. MwSt., Artikel-Nr. 901916; 12er-Pack Thieme-CME-Wertmarken, Preis: 99,- Euro inkl. MwSt., Artikel-Nr. 901917. Bitte richten Sie Bestellungen an: Georg Thieme Verlag KG, Kundenservice, Postfach 301120, 70451 Stuttgart.

Feld für CME-Wertmarke

Bitte hier Wertmarke aufkleben,

Abonnenten bitte Abonummer eintragen

Teilnahmebedingungen der zertifizierten Fortbildung

Für diese Fortbildungseinheit erhalten Sie 3 Fortbildungspunkte im Rahmen des

Fortbildungszertifikates der Ärztekammern. Hierfür:

- müssen 70\% der Fragen richtig beantwortet sein.

- muss die oben stehende Erklärung sowie die Evaluation vollständig ausgefüllt sein. Unvollständig ausgefüllte Bögen können nicht berücksichtigt werden!

- muss Ihre Abonnentennummer im entsprechenden Feld des Antwortbogens angegeben oder eine CME-Wertmarke im dafür vorgesehenen Feld aufgeklebt sein.

\begin{tabular}{|c|c|c|}
\hline Ihr Ergebnis & Sie haben & Fragen richtig beantwortet und somit \\
\hline (wird vom Verlag ausgefüllt) & bestanden und $3 \mathrm{~F}$ & dungspunkte erworben \\
\hline
\end{tabular}

Stuttgart, (Stempel/Unterschrift)

Wichtige Hinweise

Die CME-Beiträge der Notfall \& Hausarztmedizin wurden durch die Ärztekammer Nordrhein anerkannt. Die Notfall \& Hausarztmedizin ist zur Vergabe der Fortbildungspunkte für diese Fortbildungseinheit berechtigt. Diese Fortbildungspunkte der Ärztekammer Nordrhein werden von anderen zertifizierenden Ärztekammern anerkannt. Die Vergabe der Fortbildungspunkte ist nicht an ein Abonnement gekoppelt!

Bitte senden Sie den vollständig ausgefüllten Antwortbogen und einen an Sie selbst adressierten und ausreichend frankierten Rückumschlag an den Georg Thieme Verlag KG, CME, Joachim Ortleb, Postfach 301120, 70451 Stuttgart. Einsendeschluss ist der 31 . Juli 2008 (Datum des Poststempels). Die Zertifikate werden spätestens drei Wochen nach Erhalt des Antwortbogens versandt. Von telefonischen Anfragen bitten wir abzusehen. 


\section{Didaktisch-methodische Evaluation}

1 Das Fortbildungsthema kommt in meiner ärztlichen Tätigkeit

\begin{tabular}{|l|l|l|l|l|l|l|l}
\hline häufig vor & selten vor & regelmäßig vor & gar nor
\end{tabular}

2 Bezüglich des Fortbildungsthemas gab es für mich vor dem Studium dieses Beitrages

\begin{tabular}{lll} 
eine feste Gesamtstrategie $\quad$ keine Strategie noch offene Einzelprobleme: \\
\hline
\end{tabular}

3 Bezüglich des Fortbildungsthemas

fühle ich mich nach dem Studium des Beitrages in meiner Strategie bestätigt

habe ich meine Strategie geändert:

habe ich erstmals eine einheitliche Strategie erarbeitet

habe ich keine einheitliche Strategie ableiten können

4 Wurden aus der Sicht Ihrer täglichen Praxis heraus wichtige Aspekte des Themas

nicht erwähnt? Ja / Nein - wenn ja, welche:

zu knapp abgehandelt? Ja / Nein - wenn ja, welche:

überbewertet? Ja / Nein - wenn ja, welche:

5 Verständlichkeit des Beitrags

Der Beitrag ist nur für Spezialisten verständlich Der Beitrag ist auch für Nicht-Spezialisten verständlich

6 Beantwortung der Fragen

Die Fragen lassen sich aus dem Studium des Beitrages allein beantworten

Die Fragen lassen sich nur unter Zuhilfenahme zusätzlicher Literatur beantworten

7 Die Aussagen des Beitrages benötigen eine ausführlichere Darstellung

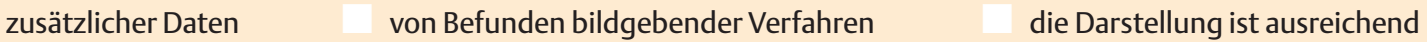

8 Wieviel Zeit haben Sie für das Lesen des Beitrages und die Bearbeitung des Fragebogens benötigt?

Minuten

\section{Fragen zur Person}

Ich bin Mitglied der Ärztekammer:

Jahr meiner Approbation:

Ich befinde mich in Weiterbildung zum:

Ich habe eine abgeschlossene Weiterbildung in (bitte Fach eintragen):

Ich bin tätig als:

Assistenzarzt

Chefarzt

Sonstiges

Oberarzt

Niedergelassener Arzt

Notfall \& Hausarztmedizin 2007; 33 (7): 381-383 